\title{
Reliability-based Interval Optimization for the Disc-Mill Cutter Machining TC17 Blisk-Tunnel
}

\author{
Nan Zhang, Guangjun Jiang*, and Jie Zhou \\ College of Mechanical Engineering, Inner Mongolia University of Technology, Hohhot, 010051, China
}

\begin{abstract}
In the paper, the sensitivity of the processing parameters for the cutting force on the disc-mill cutter machining TC17 blisk-tunnel is studied. Firstly, based on response surface methodology (RSM), a quadratic regression model between the cutting force and the processing parameters (cutting speed, feed rate per tooth, and cutting height) was established. Then, the local sensitivity of each parameter for the cutting force was calculated using the regression model, and a sensitivity curve of single parameter was acquired. Stable and unstable regions of each parameter were obtained by analyzing the sensitivity curve. Final global sensitivity of the process parameters to the cutting force was computed using the Sobol index method. The main effect, total effect, and interactive effect indexes were acquired. The results suggested that the cutting force for the disc-mill cutter machining blisk-tunnel is sensitive to the cutting speed and the feed rate per tooth. There exists a remarkable interaction between the cutting speed, feed rate per tooth, and cutting height on the cutting force. The cutting speed with [30,50], feed rate per tooth with [0.02,0.065], and cutting height with $[28,43]$ are stable regions for the cutting force.
\end{abstract}

Keywords: disc-mill cutter; cutting force; process parameters; local sensitivity analysis; global sensitivity analysis

(Submitted on September 13, 2018; Revised on October 14, 2018; Accepted on November 20, 2018)

(C) 2019 Totem Publisher, Inc. All rights reserved.

\section{Introduction}

Blisk is a novel integrated structure of blade and disk widely used in the high propulsion aero-engine. Compared with traditional blades and disk systems, blisk has the advantages of being lightweight and long-lasting and having a small amount of impulse, high efficiency, and superior reliability. Blisk is generally made of a titanium alloy with excellent high specific strength and corrosion resistance. The titanium alloy is a typical difficult-to-machine material, because it has low thermal conductivity, a small deformation coefficient, and high chemical activity. It is difficult to evacuate for the semiclosed structure of blisk; therefore, the cutting heat cannot be eliminated in time, leading to temperature rise, tool wear resistance decline, and cutting force increase. Raster modes for the ball-end mill and plunge milling for the cylindrical end mill have also been used in common milling blisk-tunnel; however, these methods have low efficiency, severe tool-wear, and high manufacturing cost. Therefore, disc-mill cutters are employed to mill blisk-tunnel. This machining can increase the material removal rate and reduce cost. The high material removal rate leads to an increase in the cutting force, so the cutting force needs to be controlled to ensure tool life and processing stability. Studying the sensitivity of machining parameters to the cutting force can enhance efficiency, extend cutter life, and reduce manufacturing costs [1-2].

Machining parameters, tool parameters, tool wear mechanisms, surface integrity, and cutting efficiency of various processing methods for titanium alloys have been researched. Some scholars have studied the influence of machining parameters on surface integrity. For instance, aiming at minimizing the surface roughness and burr formation, multiobjective optimization was made using a statistically based model and a particle swarm optimization method on micro milling TC4 by Thepsonthi et al. [3]. Some research works have focused on the effect of tool parameters on surface integrity, cutting force, and cutting heat. For example, the main tool geometry parameters that affect surface integrity of machining Ti-5Al-5Mo-5V-1Cr-1Fe were investigated by Ren et al. [4].

\footnotetext{
* Corresponding author.

E-mail address: jiang2003@imut.edu.cn
} 
Abbasi et al. investigated the influence of changing the rake angle of the PCD insert on cutting forces, tool-chip interface temperature, and tool stresses and compared the results with a TiAIN coated carbide inserted end mill utilizing FEM. The result showed that the tool rake angle has significant influence and reveals a difference in the performance for the two kind of inserts [5]. Other researchers have analyzed tool wear mechanisms of machining titanium alloys. For instance, mechanisms of cutter wear for cemented carbides (coated and uncoated) and ceramic cutter turning IMT318 and C-Ti were studied by Dearnley et al [6]. Rashid et al. revealed mechanisms of tool wear and crater formation when high speed dry cutting TC4 with uncoated carbide cutters [7].

The aforementioned research works indicate that the influence of machining parameters on surface integrity, cutting force, cutting heat, etc., have gained comprehensive attention. However, research on the sensitivity analysis of process parameters is very limited. The goal of sensitivity analysis is to identify the significant and insignificant influence factors in design variables. Sensitivity analysis has two categories: local and global sensitivity analysis. Many studies have been done on local sensitivity analysis. For example, a simple common local sensitivity analysis method was presented by Verbeke et al. [8]. Castillo et al. proposed a general approach for local sensitivity analysis, which is applied to a regression model and optimization problem [4]. Subsequently, a closed expression for local sensitivity analysis was presented by Castillo et al [9]. A hybrid local-global sensitivity analysis method was proposed and applied to a hydrologic model by Rakovec et al. Compared with the Sobol' index approach, both methods discerned comparable significant and insignificant parameters, where the hybrid method demonstrated more detailed insight with lower computational cost [10]. Local sensitivity analysis was developed based on a linear model and cannot provide accurate results when analyzing a nonlinear model. Therefore, the global sensitivity analysis was developed. The most commonly used methods of sensitivity analysis include polynomial regression, Morris screening method, Regionalized Sensitivity Analysis (RSA), general likelihood uncertainty estimation (GLUE), Fourier amplitude sensitivity (FAST), expanded Fourier amplitude sensitivity test (EFAST), and Sobol index method. Based on the Fourier amplitude sensitivity test, a new global sensitivity analysis approach was developed by Sudret et al. The two primary advantages of the approach are its robustness and high computing efficiency [11]. Li et al. proposed a new global sensitivity analysis method for systems whose inputs are independent and/or interrelated. The approach can be used to uncontrolled input variables [12]. Tahmasbi et al. applied Sobol sensitivity analysis to study temperature and force in the robotic bone drilling process [13]. To determine the most sensitive factor for the Tomgro model, the Sobol method was compared with expanded Fourier amplitude sensitivity by Vazquez-Cruz et al. [8].

In this paper, local and global sensitivity of processing parameters (cutting speed, feed rate per tooth, cutting height) for cutting force are analyzed in the process of disc-mill cutter machining TC17 blisk-tunnel. Section 2 introduces the approach of sensitivity analysis. Experimental procedures and results are given in Section 3. In Section 4, local and global sensitivity analysis of process parameters for cutting force are calculated, and stable and unstable regions of each parameter are acquired. Finally, conclusions are given in Section 5.

\section{Sensitivity Analysis}

Sensitivity means the sensitive degree of the design objective when design variables are changed. The goal of sensitivity analysis is to identify crucial and weak factors in design variables. According to the changed situation of variables and action range of sensitivity analysis, these methods can be divided into local and global sensitivity analysis. Local sensitivity analysis only examines the impact of changes of a single variable on the objective function. In contrast, global sensitivity analysis tests the total influence of several variables that are simultaneously changed and the interaction effects of variables on the objective function.

\subsection{Local Sensitivity Analysis}

In local sensitivity analysis, the differential of the output on input or output change caused by a single input variable change is regarded as the sensitivity index. Generally, local sensitivity analysis has high accuracy in a design space when calculating input-output relations around fixed point.

The degree of sensitivity reflects the gradient change of the objective function $f(x)$ to the design variable $x_{i}$. In a continuous system, if $f(x)$ is a differentiable function, its first-order sensitivity $\mathrm{S}$ can be represented as [14]:

$$
S\left(x_{i}\right)=\frac{\partial f(x)}{\partial x_{i}}
$$




\subsection{Global Sensitivity Analysis}

In global sensitivity analysis, the influence of each parameter and interaction of parameters are analyzed when several parameters simultaneously change. Global sensitivity analysis has two features:

- The variation ranges of other parameters and the influence of probability distribution are considered.

- The sensitivity of each input parameter is obtained through several input parameters varying at the same time.

Global sensitivity analysis can be applied to nonlinear and non-monotonic model. Global sensitivity analysis searches more design space for parameters and overcomes shortcomings of local sensitivity analysis, in which several parameters cannot change at the same time. Therefore, the results of global sensitivity analysis have greater adaptability. The Sobol index approach is a variance-based global sensitivity analysis. It evaluates the sensitivity of a single input and interaction of multiple inputs by computing the output variance contribution for a single input and multiple inputs [15].

If the objective function is $Y=f(x)=f\left(x_{1}, x_{2}, \cdots, x_{n}\right)$, where $x_{i}$ follows a uniform distribution in the interval [0,1], then $f(x)$ can be decomposed as follows [16-17]:

$$
f(x)=f_{0}+\sum_{i=1}^{n} f_{i}\left(x_{i}\right)+\sum_{i=1}^{n} \sum_{j=i+1}^{n} f_{i j}\left(x_{i}, x_{j}\right)+\cdots+f_{1,2, \cdots, n}\left(x_{1}, \cdots, x_{n}\right)
$$

The total variance of $f(x)$ is defined as:

$$
V=\int f^{2}(x) \mathrm{d} x-f_{0}^{2}
$$

In accordance with each decomposition in Equation (2), the partial variance of a single parameter and multiple parameters can be calculated by:

$$
\begin{gathered}
V_{i}=\int f_{i}^{2}\left(x_{i}\right) \mathrm{d} x_{i} \\
V_{i_{1} \cdots i_{s}}=\int \cdots \int f_{i_{1} \cdots i_{s}}^{2}\left(x_{i}, \cdots, x_{s}\right) \mathrm{d} x_{i_{1}} \cdots \mathrm{d} x_{i_{s}}
\end{gathered}
$$

The sensitivity parameter is defined as:

$$
S_{i_{1} \cdots i_{s}}=\frac{V_{i_{1} \cdots i_{s}}}{V}
$$

The main effect index is defined as:

$$
S_{x_{i}}=\frac{V_{x_{i}}\left(E_{x_{-i}}\left(Y \mid x_{i}\right)\right)}{V(Y)}
$$

The total effect index is defined as:

$$
S_{x_{i}}^{T}=\frac{V(Y)-V_{x_{-i}}\left(E_{x_{i}}\left(Y \mid x_{-i}\right)\right)}{V(Y)}
$$

The interactive effect index is defined as:

$$
S_{x_{i} x_{j}}=\frac{V_{x_{i} x_{j}}\left(E_{-x_{i} x_{j}}\left(Y \mid x_{i} x_{j}\right)\right)-V_{x_{i}}\left(E_{-x_{i}}\left(Y \mid x_{i}\right)\right)-V_{x_{j}}\left(E_{-x_{j}}\left(Y \mid x_{j}\right)\right)}{V(Y)}
$$


$S_{x_{i}}$ reflects the individual influence of variable $x_{i}$ on the variance of $Y . S_{x_{i}}^{T}$ displays the impact of the interaction between $x_{i}$ and the other variables on the variance of $Y$.

\section{Experiment Procedures}

\subsection{Experimental Design}

Cutting speed, feed rate per tooth, and axial or radial depth of cut are usually chosen as the control variables of milling when optimizing performance parameters. For the disc-mill cutter milling blisk-tunnel, the axial depth of cut is the width of the indexable insert. The radial depth of the cut depends on the feed rate per tooth, so the two variables cannot be chosen as the control variable. The cutting height that is the thickness of the blisk-tunnel impacts the cutting force; therefore, the cutting height is used as one of performance controlling variables. The ranges of the three performance controlling variables are listed in Table 1. An orthogonal experiment with three factors and five levels are adopted. Table 2 lists detailed parameters of 25 experiments.

Table 1. Process parameters and their levels

Table 1. Process parameters and their levels
\begin{tabular}{|c|c|c|c|}
\hline Level & $v(\mathrm{~m} / \mathrm{min})$ & $f_{c}(\mathrm{~mm} /$ tooth $)$ & $a_{w}(\mathrm{~mm})$ \\
\hline 1 & 30 & 0.020 & 28 \\
\hline 2 & 40 & 0.035 & 33 \\
\hline 3 & 50 & 0.050 & 38 \\
\hline 4 & 60 & 0.065 & 43 \\
\hline 5 & 70 & 0.080 & 48 \\
\hline
\end{tabular}

Table 2. Design of the experiment and cutting force

\begin{tabular}{|c|c|c|c|c|}
\hline \multicolumn{5}{|c|}{ Table 2. Design of the experiment and cutting force } \\
\hline No. & $v(\mathrm{~m} / \mathrm{min})$ & $f_{c}(\mathrm{~mm} /$ tooth $)$ & $a_{w}(\mathrm{~mm})$ & $F(\mathrm{~N})$ \\
\hline 1 & 30 & 0.020 & 28 & 1098.6 \\
\hline 2 & 30 & 0.035 & 33 & 1246.8 \\
\hline 3 & 30 & 0.050 & 38 & 1538.7 \\
\hline 4 & 30 & 0.065 & 43 & 1808.6 \\
\hline 5 & 30 & 0.080 & 48 & 2158.4 \\
\hline 6 & 40 & 0.020 & 33 & 1127.4 \\
\hline 7 & 40 & 0.035 & 38 & 1317.5 \\
\hline 8 & 40 & 0.050 & 43 & 1577.9 \\
\hline 9 & 40 & 0.065 & 48 & 1867.3 \\
\hline 10 & 40 & 0.080 & 28 & 2249.3 \\
\hline 11 & 50 & 0.020 & 38 & 1196.3 \\
\hline 12 & 50 & 0.035 & 43 & 1398.5 \\
\hline 13 & 50 & 0.050 & 48 & 1614.8 \\
\hline 14 & 50 & 0.065 & 28 & 1915.7 \\
\hline 15 & 50 & 0.080 & 33 & 2434.9 \\
\hline 16 & 60 & 0.020 & 43 & 1239.3 \\
\hline 17 & 60 & 0.035 & 48 & 1436.9 \\
\hline 18 & 60 & 0.050 & 28 & 1651.7 \\
\hline 19 & 60 & 0.065 & 33 & 2019.3 \\
\hline 20 & 60 & 0.080 & 38 & 2591.8 \\
\hline 21 & 70 & 0.020 & 48 & 1365.9 \\
\hline 22 & 70 & 0.035 & 28 & 1508.2 \\
\hline 23 & 70 & 0.050 & 33 & 1726.9 \\
\hline 24 & 70 & 0.065 & 38 & 2187.1 \\
\hline 25 & 70 & 0.080 & 43 & 2785.3 \\
\hline
\end{tabular}

\subsection{Experimental Design}

The workpiece material is TC17 titanium alloy. The chemical composition and mechanical properties of TC17 are listed in Tables 3 and 4, respectively. The up-milling operation for disc milling was carried out with a coolant using the complex machine tool. The disc-mill cutter with an indexable insert has 39 teeth, and the cutter diameter is $420 \mathrm{~mm}$. Square cemented carbide indexable inserts were used. Table 5 illustrates the cutter detail parameters. Figure 1 displays the machining process of the blisk-tunnel. 
Table 3. Chemical composition of TC17

\begin{tabular}{|c|c|c|}
\hline $\mathrm{Al}$ & $\mathrm{Sn}$ & $\mathrm{Zr}$ \\
\hline $4.5 \%-5.5 \%$ & $1.6 \%-2.4 \%$ & $1.6 \%-2.4 \%$ \\
\hline \hline $\mathrm{Mo}$ & $\mathrm{Cr}$ & $\mathrm{Ti}$ \\
\hline $3.5 \%-4.5 \%$ & $3.5 \%-4.5 \%$ & Balance \\
\hline
\end{tabular}

Table 4. Mechanical features of TC17 $\left(20^{\circ}\right)$
\begin{tabular}{|c|c|c|c|c|}
\hline$T_{S}(\mathrm{MPa})$ & $Y_{S}(\mathrm{MPa})$ & $E_{m}(\mathrm{GPa})$ & $E(\%)$ & $D\left(\mathrm{~g} / \mathrm{cm}^{3}\right)$ \\
\hline 1120 & 1030 & 112 & 10 & 4.68 \\
\hline
\end{tabular}

Table 5. Cutter and insert geometric parameters

\begin{tabular}{|c|c|c|c|c|}
\hline Number of teeth & Diameter $(\mathrm{mm})$ & Thickness $(\mathrm{mm})$ & Rake angle $\left(^{\circ}\right)$ & Flute length $(\mathrm{mm})$ \\
\hline 39 & 420 & 15 & 8 & 6 \\
\hline
\end{tabular}

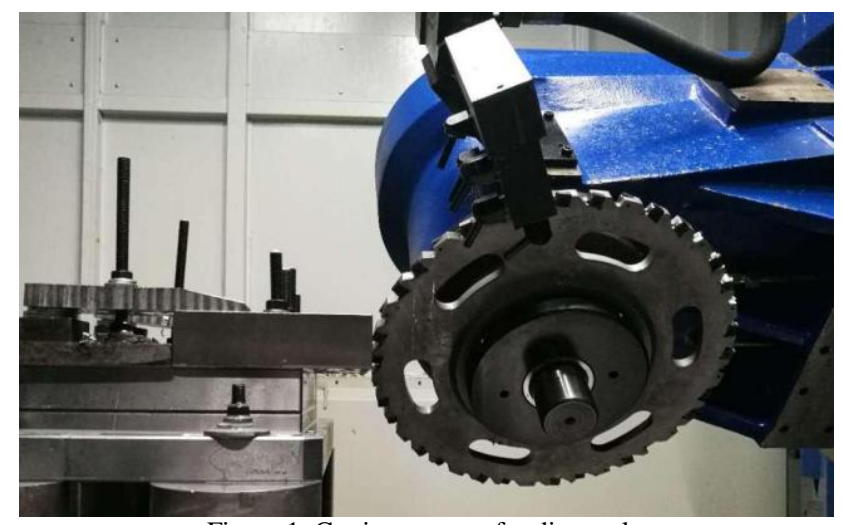

Figure 1. Cutting process for disc tools

A three coordinate dynamometer (9255B, Kistler) was fixed on the plate of the complex machine tool, and the workpiece was fixed onto the dynamometer platform using four 12-mm bolts. The cutting force signals were enlarged via a charge amplifier (5080, Kistler), which transformed using an A/D converter (PCIM-DAS1602/16, Kistler), and recorded on a computer with software (DEWESoftX2, Kistler). The data acquisition process for the cutting force is shown in Figure 2.

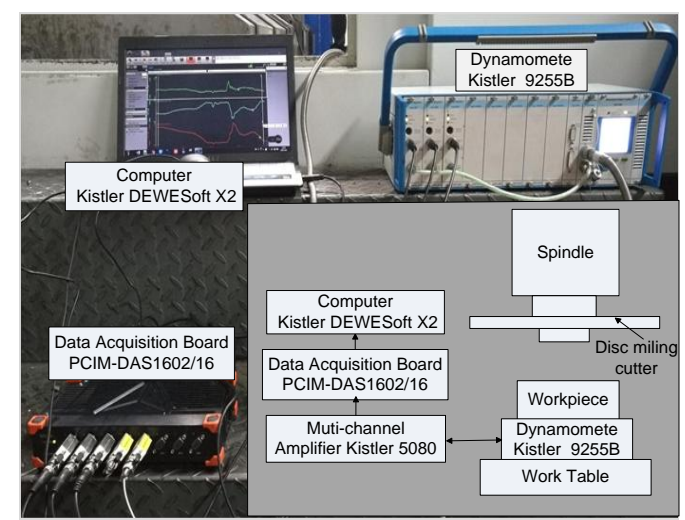

Figure 2. Data acquisition process for disc tools

\subsection{Experiment Results and Analysis}

The relationship between the cutting force and process parameters was established using the quadratic regressive model. The quadratic regressive equation between the cutting force and process parameters is expressed as [18].

$$
F=f\left(z_{i}\right)=\gamma_{0}+\sum_{i=1}^{4} \gamma_{i} z_{i}+\sum_{i=1}^{4} \sum_{j=i+1}^{4} \gamma_{i j} z_{i} z_{j}+\sum_{i=1}^{4} \gamma_{i i} z_{i}^{2}+\varepsilon
$$

Sensitivity analysis between the process parameters and cutting force is carried out based on the regressive model. 
According to the cutting force measured values of $L_{25}\left(3^{5}\right)$ orthogonal experiments, data analysis was performed on DesignExpert. The quadratic regression function between process parameters and cutting force can be written as:

$$
F=275.40+10.49 v+27014.85 f_{c}+5.93 a_{w}-124.24 v f_{c}+0.12 v a_{w}-40.44 f_{c} a_{w}-0.09 v^{2}-41625.08 f_{c}^{2}-0.12 a_{w}^{2}
$$

To verify the significance of the regressive model, a series of analysis and testing are implemented including residual analysis, variance analysis, and predicted versus measured plot [18]. Figures 3 and 4 show the residuals normal distribution and residuals run plot, respectively. It is found that data points reside around a straight line in Figure 3 and data points are scattered in Figure 4. There is no specific distribution rule for data points in Figure 4. The predicted value versus the measured value plot is displayed in Figure 5, where the nearly-line distribution indicates that the predicted values of the model are correct.

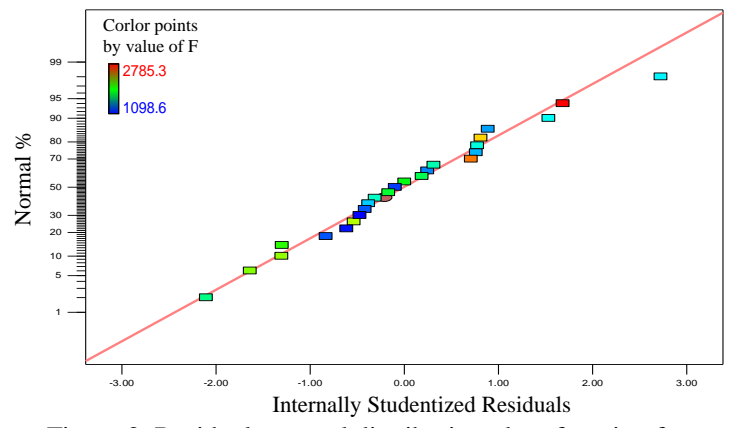

Figure 3. Residuals normal distribution plot of cutting force

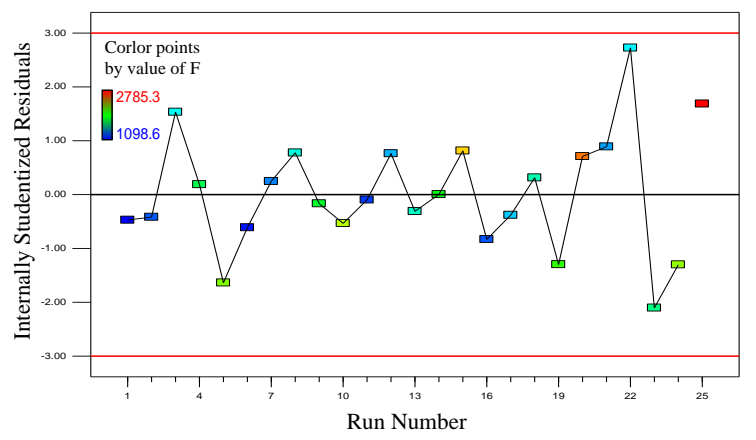

Figure 4. Residuals run plot of cutting force test results

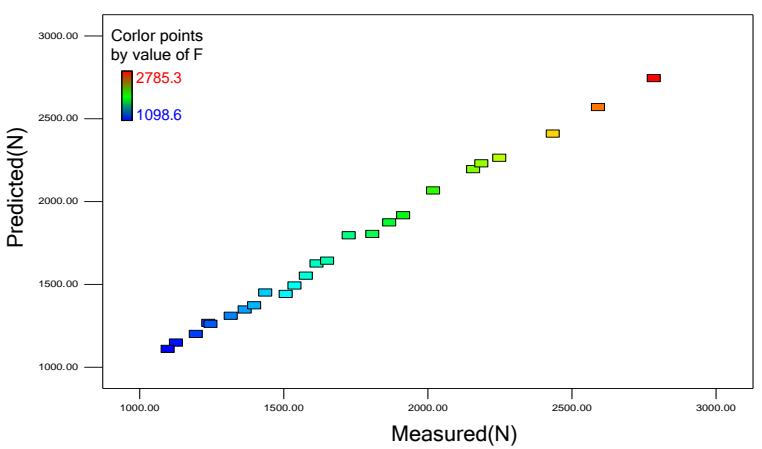

Figure 5. Predicted versus measured plot

\section{Sensitivity Analysis of Process Parameters}

\subsection{Local Sensitivity Analysis of Each Process Parameter}

Local sensitivity means the sensitive degree of the response variable when only a single input variable changes. According to the sensitivity definition, the sensitive degree model of the cutting force to machining parameters is represented as Equation (12) [19]. 


$$
\left\{\begin{array}{l}
S_{F}^{v}=|8.77-0.18 v| \\
S_{F}^{f_{c}}=\left|4659.49-83250.16 f_{c}\right| \\
S_{F}^{a_{w}}=\left|9.81-0.24 a_{w}\right|
\end{array}\right.
$$

The cutting force model was obtained by experiment. The initial process parameters are discrete points. Hence, the values of $v, f_{c}$, and $a_{w}$ in Equation (12) are set as average value of process parameters with $\bar{v}=50 \mathrm{~m} / \mathrm{min}$, $\overline{f_{c}}=0.05 \mathrm{~mm} /$ tooth , and $\overline{a_{w}}=38 \mathrm{~mm}$.

The sensitivity curves of the cutting speed, feed rate per tooth, and cutting height for the cutting force are shown in Figures 6,7, and 8, respectively.

The local sensitive degree of the cutting force to process parameters reflects the change rate of the cutting force in a certain process parameter range. Therefore, optimizing the interval of each process parameter can be achieved according to the sensitivity curve.

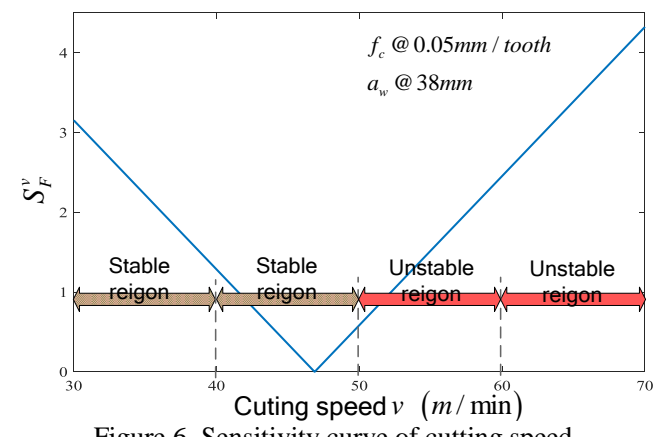

Figure 6. Sensitivity curve of cutting speed

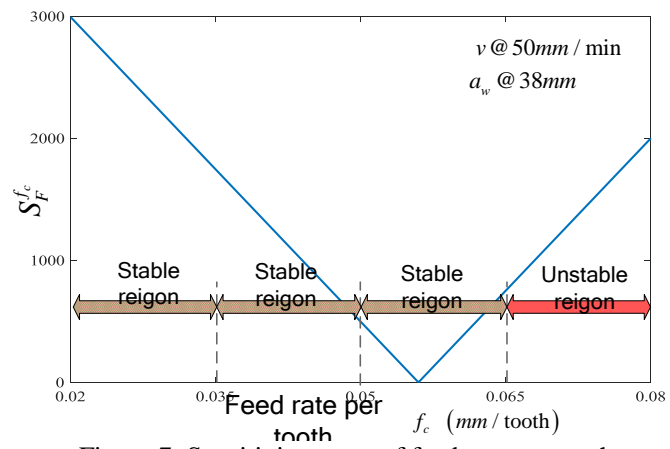

Figure 7. Sensitivity curve of feed rate per tooth

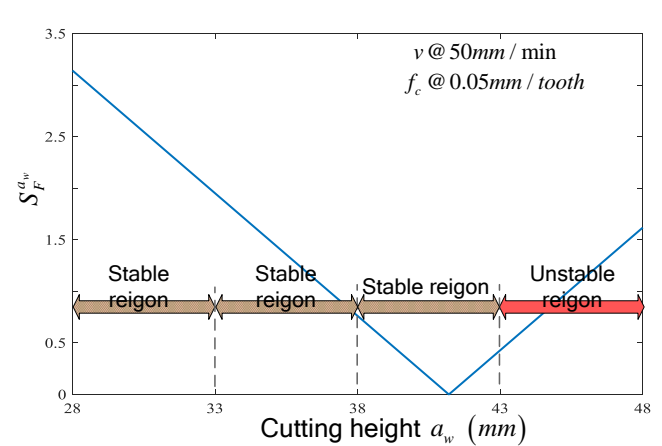

Figure 8. Sensitivity curve of cutting height

\subsection{Stable and Unstable Region Analysis of Process Parameter}

The stable region of process parameters is insensitive to the variation of process parameters, while the unstable region of process parameters is sensitive to the variation of process parameters in the range [19].

The orthogonal test has $n$ factors $\left(N_{1}, N_{2}, \cdots, N_{n}\right)$ with $m$ levels $\left(M_{1}, M_{2}, \cdots, M_{m}\right)$. The approach of division stable and unstable region of process parameters is written as [19]:

- According to the single-parameter sensitivity curve, the amplitude change of sensitivity for each factor is calculated in the interval of $\left[M_{1}, M_{2}\right],\left[M_{2}, M_{3}\right], \cdots,\left[M_{m-1}, M_{m}\right]$, which is denoted as $A_{1}, A_{2}, \cdots, A_{m-1}$.

- Compute the average value of $m-1$ amplitude changes of sensitivity. The average value is expressed as $A_{0}$. If $A_{j}(j=1,2, \cdots, m-1)>A_{0},\left[M_{j}, M_{j+1}\right]$ is defined as an unstable region. If not, the interval is a stable region. 
Based on the local sensitivity curve, the stable and unstable region of the cutting speed, feed rate per tooth, and cutting height can be obtained. The stable and unstable regions of the process parameters are outlined in Table 6 . The stable regions are: cutting speed with [30,50], feed rate per tooth with [0.02,0.065], and cutting height with $[28,43]$. The unstable region of the cutting speed, feed rate per tooth, and cutting height are $[50,70],[0.065,0.08]$, and $[43,48]$, respectively.

Table 6. Stable and unstable regions of process parameters

\begin{tabular}{|c|c|c|}
\hline Process parameters & Stable region & Unstable region \\
\hline$v$ & {$[30,50]$} & {$[50,70]$} \\
\hline$f_{c}$ & {$[0.02,0.065]$} & {$[0.065,0.08]$} \\
\hline$a_{w}$ & {$[28,43]$} & {$[43,48]$} \\
\hline
\end{tabular}

\subsection{Global Sensitivity Analysis of Process Parameter}

Local sensitivity can perfectly reflect the range of response variables when only one input variable changes, but it does not synthetically reflect the sensitive degree of the input variable. Global sensitivity can do so. The Sobol index method is adopted to calculate global sensitivity in the paper. The input of the Sobol method follows a uniform distribution in the interval of $[0,1]$ [3]. Thus, the input variables need to be standardized. The process parameters and cutting force are normalized to $y_{1}, y_{2}, y_{3}$, and $F^{\prime}$, as shown in Table 7 . The second-order regression model of the cutting force becomes:

$$
F^{\prime}=2.6 \times 10^{-3}+0.012 y_{1}+0.24 y_{2}+0.11 y_{3}+0.19 y_{1} y_{2}+0.049 y_{1} y_{3}-7.77 \times 10^{-3} y_{2} y_{3}+0.050 y_{1}^{2}+0.41 y_{2}^{2}-0.087 y_{3}^{2}
$$

Table 7. Standardized data

\begin{tabular}{|c|c|c|c|c|}
\hline No. & $y_{1}$ & $y_{2}$ & $y_{3}$ & $F^{\prime}$ \\
\hline 1 & 0 & 0 & 0 & 0.0000 \\
\hline 2 & 0 & 0.25 & 0.25 & 0.0879 \\
\hline 3 & 0 & 0.5 & 0.5 & 0.2609 \\
\hline 4 & 0 & 0.75 & 0.75 & 0.4209 \\
\hline 5 & 0 & 1 & 1 & 0.6283 \\
\hline 6 & 0.25 & 0 & 0.25 & 0.0171 \\
\hline 7 & 0.25 & 0.25 & 0.5 & 0.1298 \\
\hline 8 & 0.25 & 0.5 & 0.75 & 0.2842 \\
\hline 9 & 0.25 & 0.75 & 1 & 0.4557 \\
\hline 10 & 0.25 & 1 & 0 & 0.6822 \\
\hline 11 & 0.5 & 0 & 0.5 & 0.0579 \\
\hline 12 & 0.5 & 0.25 & 0.75 & 0.1778 \\
\hline 13 & 0.5 & 0.5 & 1 & 0.3060 \\
\hline 14 & 0.5 & 0.75 & 0 & 0.4844 \\
\hline 15 & 0.5 & 1 & 0.25 & 0.7923 \\
\hline 16 & 0.75 & 0 & 0.75 & 0.0834 \\
\hline 17 & 0.75 & 0.25 & 1 & 0.2006 \\
\hline 18 & 0.75 & 0.5 & 0 & 0.3279 \\
\hline 19 & 0.75 & 0.75 & 0.25 & 0.5459 \\
\hline 20 & 0.75 & 1 & 0.5 & 0.8853 \\
\hline 21 & 1 & 0 & 1 & 0.1585 \\
\hline 22 & 1 & 0.25 & 0 & 0.2428 \\
\hline 23 & 1 & 0.5 & 0.25 & 0.3725 \\
\hline 24 & 1 & 0.75 & 0.5 & 0.6453 \\
\hline 25 & 1 & 1 & 0.75 & 1.0000 \\
\hline
\end{tabular}

The sensitivity analysis procedure of the Sobol index method is shown below [16,20]:

- Define upper and lower bounds of the input variable of $y_{i}$.

- Generate the value range of the input set.

- Take the cutting force model of Equation (13) into the input set.

- Calculate the sensitivity modulus of the main effect, total effect, and interactive effect using Equations (7), (8), and (9), respectively.

- Analyze the sensitivity modulus. 
Table 8 gives the main and total effects of the sensitivity index of the cutting speed, feed rate per tooth, and cutting height for cutting force. The main effect sensitivity indexes are: cutting speed with 0.3258 , feed rate per tooth with 0.5860 , and cutting height with 0.0451 . The total effect sensitivity modulus of the cutting speed, feed rate per tooth, and cutting height are $0.3403,0.6020$, and 0.0510 , respectively. The sensitivity modulus of the feed rate per tooth and cutting speed for cutting force are fairly larger, while the cutting height is relatively lower. This indicates that the feed rate per tooth and cutting speed have a greater impact on the cutting force, whereas the cutting force is not sensitive to the cutting height. Meanwhile the difference between the main effect and total effect for the cutting speed and feed rate per tooth are bigger, showing that the cutting speed and feed rate per tooth interact with other process parameters. The difference for the cutting height is comparatively smaller, and it can be found that the interaction between the cutting height and the other process parameters is not significant.

Table 8. Sensitivity index of process parameters for cutting force

\begin{tabular}{|c|c|c|c|}
\hline Process parameters & Main effect $S_{y_{i}}$ & Total effect $S_{y_{i}}^{T}$ & Difference value \\
\hline$y_{1}$ & 0.3258 & 0.3403 & 0.0145 \\
\hline$y_{2}$ & 0.5860 & 0.6020 & 0.0160 \\
\hline$y_{3}$ & 0.0451 & 0.0510 & 0.0059 \\
\hline
\end{tabular}

\section{Conclusions}

Global sensitivity and single-parameter sensitivity of the process parameters for the disc-mill cutter machining blisk-tunnel are analysed in the paper. The stable and unstable regions of process parameters are determined. Conclusions can be drawn as follows:

- The cutting force for the disc-mill cutter machining blisk-tunnel is sensitive to the cutting speed and feed rate per tooth. There exists a remarkable interaction between cutting speed, feed rate per tooth, and cutting height on cutting force.

- The local sensitivity of all parameters is firstly declined, and the sensitivity is increased once the parameters are larger than the given size.

- For the cutting force of the disc-mill cutter machining blisk-tunnel, the stable regions are: cutting speed with $[30,50]$, feed rate per tooth with $[0.02,0.065]$, and cutting height with $[28,43]$.

\section{Acknowledgements}

This work was partially supported by the National Natural Science Foundation of China (No. 71761030).

\section{References}

1. P. B. Zhao and Y. Shi, "Robust Control of the A-Axis with Friction Variation and Parameters Uncertainty in Five-Axis CNC Machine Tools," Archive Proceedings of the Institution of Mechanical Engineers Part C Journal of Mechanical Engineering Science 1989-1996, Vol. 228, No. 14, pp. 2545-2556, September 2014

2. P. B. Zhao, Y. Shi, and J. Huang, "Proportional-Integral based Fuzzy Sliding Mode Control of the Milling Head," Control Engineering Practice, Vol. 3, No. 5, pp. 1-13, August 2016

3. T. Thepsonthi and T. Özel, "Multi-Objective Process Optimization for Micro-End Milling of Ti-6Al-4V Titanium Alloy," International Journal of Advanced Manufacturing Technology, Vol. 63, No. 12, pp. 903-914, December 2012

4. P. A. Dearnley and A. N. Grearson, "Evaluation of Principal Wear Mechanisms of Cemented Carbides and Ceramics used for Machining Titanium Alloy IMI 318," Metal Science Journal, Vol. 2, No. 1, pp. 47-58, July 2013

5. J. Ren, J. Zhou, and J. Zeng, "Analysis and Optimization of Cutter Geometric Parameters for Surface Integrity in Milling Titanium Alloy Using a Modified Grey-Taguchi Method," Proceedings of the Institution of Mechanical Engineers, Part B: Journal of Engineering Manufacture, Vol. 230, No. 11, pp. 2114-2128, September 2015

6. S. A. Abbasi and P. Feng, "Evaluating the Effect of PCD and TiAIN Coated Carbide End Mill's Rake Angle on Machinability of Titanium Alloy TÃ-6AI-4V," Advanced Materials Research, Vol. 1025-1026, pp. 564, September 2014

7. R. A. R. Rashid, S. Palanisamy, S. Sun, and M. S. Dargusch, "Tool Wear Mechanisms Involved in Crater Formation on Uncoated Carbide Tool When Machining Ti6al4v Allo," International Journal of Advanced Manufacturing Technology, Vol. 83, No. 9, pp. 1457-1465, April 2016

8. M. A. Vazquez-Cruz, R. Guzman-Cruz, I. L. Lopez-Cruz, O. Cornejo-Perez, I. Torres-Pacheco, and R. G. Guevara-Gonzalez, "Global Sensitivity Analysis by Means of Efast and Sobol' Methods and Calibration of Reduced State-variable Tomgro Model Using Genetic Algorithms," Computers and Electronics in Agriculture, Vol. 100, pp. 1-12, January 2014

9. E. Castillo, A. S. Hadi, A. Conejo, and A. Fernández-Canteli, "A General Method for Local Sensitivity Analysis with 
Application to Regression Models and Other Optimization Problems," Technometrics, Vol. 46, No. 4, pp. 430-444, November 2004

10. A. Saltelli and S. Tarantola, "A Quantitative Model-Independent Method for Global Sensitivity Analysis of Model Output," Technometrics, Vol. 41, No. 1, pp. 39-56, 1999

11. B. Sudret, "Global Sensitivity Analysis Using Polynomial Chaos Expansions," Reliability Engineering and System Safety, Vol. 93, No. 7, pp. 964-979, July 2008

12. G. Li, H. Rabitz, P. E. Yelvington, O. O. Oluwole, F. Bacon, and C. E. Kolb, "Global Sensitivity Analysis for Systems with Independent and/or Correlated Inputs," Journal of Physical Chemistry, Vol. 2, No. 6, pp. 7587-7589, May 2010

13. V. Tahmasbi, M. Ghoreishi, and M. Zolfaghari, "Sensitivity Analysis of Temperature and Force in Robotic Bone Drilling Process Using Sobol Statistical Method," Biotechnology and Biotechnological Equipment, Vol. 9, pp. 1-12, November 2017

14. G. Verbeke, G. Molenberghs, H. Thijs, E. Lesaffre, and M. G. Kenward, "Sensitivity Analysis for Nonrandom Dropout: A Local Influence Approach," Biometrics, Vol. 57, No. 1, pp.1-7, March 2001

15. C. Z. Li and S. Mahadevan, "An Efficient Modularized Sample-based Method to Estimate the First-Order Sobol' Index," Reliability Engineering and System Safety, Vol. 153, pp. 110-121, September 2016

16. F. Pianosi, K. Beven, J. E. Freer, J. W. Hall, J. Rougier, D. B. Stephenson, et al., "Sensitivity Analysis of Environmental Models: A Systematic Review with Practical Workflow," Environmental Modelling and Software, Vol. 79, No. C, pp. 214-232, May 2016

17. R. Rosolem, H. V. Gupta, W. J. Shuttleworth, and X. Zeng, "A Fully Multiple-Criteria Implementation of the Sobol' Method for Parameter Sensitivity Analysis," Journal of Geophysical Research Atmospheres, Vol. 117, No. D7, pp. 287-298, April 2015

18. M. J. Anderson and P. J. Whitcomb, "Rsm Simplified: Optimizing Processes Using Response Surface Methods for Design of Experiments Second Edition," Crc Press, 2016

19. T. Yu, Y. Shi, X. He, C. Kang, B. Deng, and S. Song, "Optimization of Parameter Ranges for Composite Tape Winding Process based on Sensitivity Analysis," Applied Composite Materials, Vol. 59, No. 2, pp. 1-16, August 2016

20. B. Zhou, L. Wang, Y. Liu, and Y. Hu, "Analysis of Factor Sensitivity in Brake Friction and Wear Performance based on the Sobol Method," Journal of the Brazilian Society of Mechanical Sciences and Engineering, Vol. 40, No. 7, pp. 345, July 2018 